\title{
Beziehung zwischen mittels Dualenergie-Röntgenabsorptiometrie bestimmter Körperzusammensetzung und Fruchtbarkeit von Jungsauen
}

\begin{abstract}
Title of the paper: Relationship between body composition measured by dual energy x-ray absorptiometry and reproduction performance in gilts

This study was conducted to evaluate the relationship between body composition and reproduction traits in gilts. A number of 176 gilts of different genotypes were categorized in three different groups. Group 0 consists of gilts that were disqualified for breeding according performance test results or showed no occurrence of oestrus. Group 1 consists of all gilts, which farrowed after an artificially insemination. Group 2 was composed of gilts that were artificially inseminated but did not give birth to a litter.

Body composition was determined in vivo using dual energy x-ray absorptiometry at a live weight of about 90 $\mathrm{kg}$. Initial reproduction data from group 1 were also evaluated.

Results of the performance test showed considerable variation among the different groups. Group 1 was significantly heavier and the daily gain was significantly higher than in group 0 . However, results of breeding index and value were clearly below the results of the other two groups. The body fat content was significantly higher in gilts with a litter (>1.65 absolutely) than in gilts without a litter (group 2) and in disqualified gilts (group 0). Group 2 gilts showed the smallest body fat mass. However, the relation between DXA fat percentage and litter size for group 1 showed in tendency that the litter size might decrease with an increasing fat content of gilts. Therefore, an optimum body fat content for conventional breeding gilts of European or American origin needs to be determined for a maximum fertility.
\end{abstract}

Key Words: DXA, body composition, reproduction performance, gilts

\section{Zusammenfassung}

Ziel dieser Studie war es, die Beziehung zwischen der Körperzusammensetzung und der Fruchtbarkeit von Jungsauen zu untersuchen. 176 Jungsauen verschiedener Genotypen wurden drei Gruppen zugeordnet. Gruppe 0 setzte sich aus Tieren zusammen, die entweder aufgrund einer durchgeführten Eigenleistungsprüfung zuchtuntauglich beurteilt wurden oder keine Rauscheerscheinungen zeigten und somit nicht belegt werden konnten. Gruppe 1 wurde von Jungsauen mit einem ersten Wurf gebildet und in Gruppe 2 befanden sich Jungsauen, die besamt wurden, jedoch keinen ersten Wurf bekamen.

Von allen Tieren wurde mit Erreichen eines Lebendgewichts von ca. 90 kg eine In-vivo-Ganzkörperanalyse mit Hilfe der Dualenergie-Röntgenabsorptiometrie (DXA) durchgeführt. Außerdem wurden von allen Jungsauen der Gruppe 1 Wurfdaten erfasst.

Ergebnisse der Eigenleistungsprüfung zeigten zum Teil beträchtliche Unterschiede zwischen den einzelnen Gruppen. Tiere der Gruppe 1 hatten signifikant höhere Tageszunahmen als die der Gruppe 0. Jedoch lagen die Werte für Zuchtindex und -wert unter denen der zwei anderen Gruppen. Der Gesamtkörperfettgehalt war bei Jungsauen mit Wurf signifikant höher (>1,65 \% absolut) als bei Jungsauen ohne Wurf (Gruppe 2) bzw. zuchtuntauglichen Jungsauen (Gruppe 0). Die Jungsauen der Gruppe 2 (ohne Wurf) wiesen die niedrigsten Körperfettgehalte aller drei Gruppen auf. Innerhalb der Gruppe 1 zeigte die Beziehung zwischen DXA-Fett (\%) und der Wurfgröße jedoch tendenziell, dass mit steigendem Körperfettgehalt der Jungsauen eine Verminderung der Wurfgröße verbunden sein kann. Folglich ist für eine maximale Fruchtbarkeitsleistung von Jungsauen konventioneller Schweinerassen ein optimaler Körperfettgehalt anzustreben.

Schlüsselwörter: DXA, Ganzkörperzusammensetzung, Fruchtbarkeit, Jungsauen 


\section{Einleitung}

Das Zuchtziel in der Schweineproduktion richtet sich vor allem nach den Wünschen des Verbrauchers, das heißt reduzierte Fettanteile bei gleichzeitig erhöhtem Muskelfleischanteil werden bevorzugt. Es stellt sich die Frage, ob die Selektion auf immer magerere Tiere Fruchtbarkeitsprobleme bei Sauen (speziell Jungsauen) hervorruft. Studienergebnisse hierzu sind kontrovers. Einige Autoren stellten fest, dass Alter, Gewicht und Körperzusammensetzung während der ersten Aufzuchtphase nicht mit der Wurfgröße in Verbindung stehen (NEWTON and MAHAN, 1993; ROZEBOOM et al., 1996; SCHNURRBUSCH, 2005). Untersuchungen von WÄHNER et al. (1993, 1995) zeigten jedoch anhand von Messungen der Rückenspeckdicke, dass sich in bestimmten Grenzen ein höherer Fettgehalt im Tierkörper positiv auf die Ovaraktivität und dementsprechend auf die Wurfgröße auswirkt.

$\mathrm{Zu}$ anderen Fruchtbarkeitsparametern von Jungsauen gibt es ebenfalls unterschiedliche Aussagen. Einerseits wurden weder dem Alter und der Körperzusammensetzung von Jungsauen große Auswirkungen auf die Produktivität der Sauen beigemessen (ROZEBOOM et al., 1996), noch wurde das Eintreten der Geschlechtsreife mit dem Erreichen einer bestimmten Körperzusammensetzung in Zusammenhang gebracht (ROZEBOOM et al., 1995). Andererseits wird die Aussage getroffen, dass Jungsauen mit einem höheren Gesamtkörperfettanteil früher in Rausche kommen als Tiere mit einem geringeren Körperfettgehalt (GAUGHAN et al., 1997). Eine ähnliche Aussage trafen auch NEWTON and MAHAN (1993), die eine geringere Konzeptionsrate bei Jungsauen mit niedrigerem Körpergewicht feststellten als bei Sauen mit höherem Gewicht.

Mit Hilfe der Dualenergie-Röntgenabsorptiometrie (DXA) ist es inzwischen möglich, nicht nur einzelne Merkmale des Tierkörpers, wie zum Beispiel Gewicht oder Rückenspeckdicke am lebenden Tier zu messen, sondern die gesamte Körperzusammensetzung in vivo zu erfassen. In vielen vorangegangenen Untersuchungen hat sich bereits gezeigt, dass DXA sehr gut geeignet ist, die Körperzusammensetzung beim Schwein in vivo zu bestimmen (BÖTTINGER, 2005; KOO et al., 2002; LUKASKI et al., 1999; MITCHELL et al., 1996; 1998; 2000; 2002; MITCHELL and SCHOLZ, 1997; SCHOLZ, 2002; SCHOLZ et al., 2002; SVENDSEN et al., 1993).

In der vorliegenden Studie soll untersucht werden, welche Beziehung zwischen dem Gesamtkörperfettgehalt (ermittelt aus DXA) und der Fruchtbarkeit von Jungsauen besteht.

\section{Tiere}

\section{Material und Methoden}

Der Gesamtumfang der untersuchten Tiergruppe betrug 176 Jungsauen der Rassen Deutsches Edelschwein (DE), Deutsche Landrasse (DL), Duroc (Du), Hampshire (Ha), Pietrain (Pi), Schwäbisch-Hällisches Landschwein (SH) und Kreuzungstiere der Rassen DL*DE, Pi*Du, Pi*Ha (Tab. 1). Mit Erreichen eines Lebensalters von ca. 180 Tagen und eines Lebendgewichts von ca. $90 \mathrm{~kg}$ wurden bei den Zuchttieren eine Leistungsprüfung und Zuchtwertschätzung vom Landeskuratorium der Erzeugerringe für tierische Veredelung (LKV) in Bayern sowie eine Ganzkörperanalyse mittels Dualenergie-Röntgenabsorptiometrie (DXA) durchgeführt. Das durchschnittliche Gewicht der Sauen betrug 95,6 kg bei einer Gewichtsspanne von 74 bis $130 \mathrm{~kg}$. Das Alter lag zwischen 148 und 230 Lebenstagen. Alle untersuchten Tiere stammten vom Lehr- und 
Versuchsgut der LMU München in Oberschleißheim und wurden im Zeitraum von Juni 2000 bis März 2006 gescannt. Entsprechend des Geburtsdatums wurden sechs Geburtsjahresklassen gebildet.

Tabelle 1

Genotypen und Tierzahlen aller untersuchten Jungsauen in Abhängigkeit von der Sauengruppe (Different genotypes and number of analysed gilts depending on sow group)

\begin{tabular}{lcccc}
\hline Genotypen & $\begin{array}{c}\text { Tierzahl } \\
(\mathbf{n = 1 7 6})\end{array}$ & $\begin{array}{c}\text { Gruppe } \\
\mathbf{0}(\mathbf{n}=\mathbf{3 5})\end{array}$ & $\begin{array}{c}\text { Gruppe } \\
\mathbf{1}(\mathbf{n}=\mathbf{1 0 3})\end{array}$ & $\begin{array}{c}\text { Gruppe } \\
\mathbf{2}(\mathbf{n}=\mathbf{3 8})\end{array}$ \\
\hline $\mathrm{DE}$ & 9 & & 8 & 1 \\
$\mathrm{DL}$ & 84 & 19 & 46 & 19 \\
$\mathrm{Du}$ & 26 & 3 & 12 & 11 \\
$\mathrm{Ha}$ & 2 & & 2 & 3 \\
$\mathrm{Pi}$ & 24 & 7 & 14 & 1 \\
$\mathrm{SH}$ & 4 & & 3 & 1 \\
$\mathrm{DL} \mathrm{x} \mathrm{DE}$ & 8 & 2 & 5 & 1 \\
Pi x Du & 5 & 1 & 3 & 1 \\
Pi x Ha & 14 & 3 & 10 & \\
\hline
\end{tabular}

DE = Deutsches Edelschwein, DL = Deutsche Landrasse, $\mathrm{Du}=$ Duroc,

$\mathrm{Ha}=$ Hampshire, $\mathrm{Pi}$ = Pietrain, $\mathrm{SH}=$ Schwäbisch-Hällisches Landschwein

Gruppe 0 = zuchtuntauglich oder ohne Rauscheerscheinungen [without mating]; Gruppe 1 = zuchttauglich, mit erstem Wurf [with litter]; Gruppe 2 = zuchttauglich, ohne ersten Wurf [mated, without litter]

\section{DXA-Analyse}

Die Zuchtsauen wurden unter Verwendung eines DPX-IQ Scanners der Firma GE Lunar (Madison, Wisconsin, USA) untersucht. Die Untersuchung fand maximal drei Tage vor oder nach der LKV-Prüfung statt. Gleichzeitig wurde jedes Tier gewogen und die Lebendtageszunahmen (LTZ) ermittelt. Für die DXA-Messung wurden die Schweine zunächst sediert (intramuskuläre Injektion einer Kombination aus Azaperon (Stresnil ${ }^{\circledR}$, JANSSEN-CILAG GmbH) 1,2 mg/kg Körpergewicht [KGW] und Ketaminhydrochlorid (Ursotamin ${ }^{\circledR}$, Serumwerk Bernburg AG) $10 \mathrm{mg} / \mathrm{kg} \mathrm{KGW}$ ) und anschließend in Narkose gelegt (intravenöse Injektion von Ketaminhydrochlorid (Ursotamin ${ }^{\circledR}$ Serumwerk Bernburg AG) 20-25 mg/kg KGW). Das narkotisierte Tier wurde in Bauchlage mit ausgestreckten Vorder- und Hintergliedmaßen auf dem DXA-Tisch gelagert, so dass eine Ganzkörperanalyse (Softwaremodus ,adult normal“) durchgeführt werden konnte. Ermittelt wurden Fettgewebe (g, \%), Magerweichgewebe (g), Knochenmineralgehalt $(\mathrm{g})$ und Knochenmineraldichte $\left(\mathrm{g} / \mathrm{cm}^{2}\right)$.

Die Dualenergie-Röntgenabsorptiometrie basiert auf einem Drei-Komponenten-Modell für die Körperzusammensetzung (Abb. 1). Das Modell setzt sich aus Fettgewebe, Magerweichgewebe (Muskulatur, Bindegewebe, innere Organe, Knorpel) und Knochenmineralstoffen zusammen.

Mit Hilfe von polyenergetischen Röntgenstrahlen kann die gewebliche Zusammensetzung sowohl in ausgewählten Körperregionen als auch im Gesamtkörper bestimmt werden (MITCHELL and SCHOLZ, 2001; PIETROBELLI et al., 1996).

Das DXA-Verfahren beim GE Lunar DPX-IQ beruht auf der Nutzung von zwei Photonenenergieniveaus mit $38 \mathrm{keV}$ und $70 \mathrm{keV}$. Beim Durchdringen des Tierkörpers werden sowohl die niedrig- als auch die hochenergetischen Röntgenstrahlen unterschiedlich abgeschwächt. Das Ausmaß der Abschwächung ist abhängig von der in diesem Bereich vorhandenen Gewebeart. Jedem Gewebe kann ein Röntgenschwächungskoeffizient (R-Wert) zugeordnet werden (PIETROBELLI et al., 1996), der unabhängig von der Gewebedicke ist. Der R-Wert für Fett $(\sim 1,2)$ und Magerweichgewebe $(\sim 1,35)$ 
ist deutlich niedriger als der für Knochen $(>2,8)$. Aufgrund dieser Unterschiede wird für jeden Bildpunkt (Pixel) zunächst zwischen Weichgewebe und knochenmineralhaltigem Gewebe unterschieden. Allein für Pixel, die kein Knochenmineral enthalten, wird die Weichgewebezusammensetzung direkt ermittelt. Zusätzlich werden der Fettund Magerweichgewebegehalt für die Knochenmineral enthaltenden Pixel in Abhängigkeit vom umgebenden Weichgewebe interpoliert.

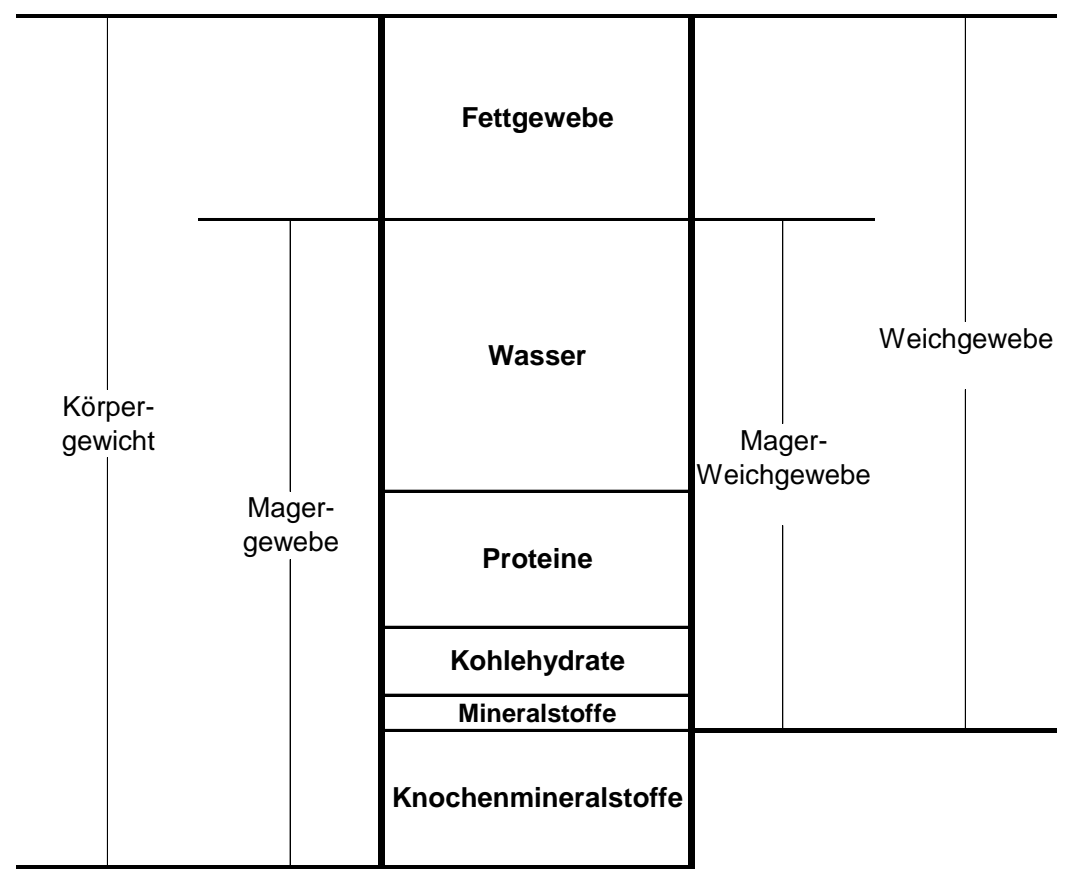

Abb. 1: Modell der Körperzusammensetzung nach PIETROBELLI (96) [Body composition model modified after PIETROBELLI (96)]

\section{LKV-Prüfung}

Alle Jungsauen wurden durch einen neutralen Tester auf Zuchttauglichkeit mit einem vom Ausschuss für Leistungsprüfung und Zuchtwertfeststellung beim Schwein zugelassenen Ultraschallgerät (Piglog 05) überprüft (MÜLLER und POLTEN, 2004). Folgende Daten wurden im Rahmen der LKV-Prüfung ermittelt: Speckdicke (Speckmaß B), Muskeldicke (beides mittels Ultraschall), Magerfleischanteil, Fundament-, Bemuskelungs- und Exterieurnote, Zitzenanzahl, Selektionsindex, Zuchtwert, Gewicht und Tageszunahmen.

\section{Fruchtbarkeitsdaten}

Bei allen Jungsauen wurden Fruchtbarkeitsdaten wie Besamungsdatum, Abferkeldatum, Anzahl geborener, aufgezogener und totgeborener Ferkel, sowie die Gesamtferkelverluste des ersten Wurfes erfasst.

Aufgrund der LKV- und Betriebsdaten wurden drei Sauengruppen gebildet. Die Gruppe 0 (Tab. 1, 2), umfasste alle Sauen, die nach der Eigenleistungsprüfung nur eine geringe Zuchttauglichkeit aufwiesen und somit von der Zucht ausgeschlossen wurden, zusätzlich alle Jungsauen, die zwar zuchttauglich waren, aber keine Rauscheerscheinungen zeigten und somit nicht belegt werden konnten. Die Gruppe 1, umfasste alle Sauen die zuchttauglich waren, ein Deckdatum und einen ersten Wurf aufwiesen; die 
Gruppe 2, umfasste alle Sauen die zur Zucht zugelassen wurden, ein Deckdatum aufwiesen, aber aufgrund fehlender Konzeption keinen ersten Wurf aufwiesen (Tab. 1).

Tabelle 2

Zuchtuntauglichkeitsursachen für Jungsauen der Gruppe 0 (Reasons for disqualified gilts in group 0)

\begin{tabular}{lc}
\hline Anzahl Tiere & 35 \\
\hline keine Rausche & 20 \\
Zunahmen $(<430 \mathrm{~g})$ & 1 \\
Fundament & 1 \\
Bemuskelung & 0 \\
Exterieur & 0 \\
Zitzenanzahl & 9 \\
Selektionsindex $(<80)$ & 7 \\
\hline
\end{tabular}

\section{Haltung und Fütterung}

Die Fütterung und Haltung war bei allen potentiellen Jungsauen gleich. Bis zum Erreichen des Absetzalters von vier Wochen verblieben die Ferkel in mit Stroh eingestreuten Abferkelbuchten. Anschließend wechselten sie in einen Flatdeckstall mit Vollspaltenboden aus Kunststoff. Mit dem Erreichen eines Körpergewichtes von 25 - 30 kg wurden die Tiere in einen Außenklimastall (Tiefstreustall mit einer über Stufen erreichbaren erhöht liegenden Fläche aus Betonspaltenboden) umgestallt. Dort verblieben sie bis zur Zuchtreife (zwischen 90 - 100 kg Körpergewicht). Mit Erreichen der Zuchtreife wurden die Jungsauen der Gruppe 1 und 2 (und teilweise der Gruppe 0) in das Deckzentrum mit Gruppenhaltung (5-8 Tiere/Gruppe) umgestallt und nach natürlichem Eintritt der Rausche zweimal im Abstand von 12 h künstlich besamt. Die Jungsauen der Gruppe 0, die in das Deckzentrum umgestallt wurden, zeigten keine Rauscheanzeichen und wurden nicht besamt.

Tabelle 3

Zusammensetzung der einzelnen Futtermischungen (Composition of the different diets)

\begin{tabular}{lccc}
\hline $\begin{array}{l}\text { Inhaltstoffe } \\
(\%)\end{array}$ & $\begin{array}{c}\text { Schauma Früh } \\
\text { (Fa. Schaumann) }\end{array}$ & $\begin{array}{c}\text { Ferkelfutter } \\
\text { (LVG) }\end{array}$ & $\begin{array}{c}\text { Niedertragende } \\
\text { Sauen (LVG) }\end{array}$ \\
\hline Gerste & 34 & 21 & 48,7 \\
Weizen & 21 & 44 & 6 \\
Hafer & & & 37 \\
Mais & 15 & 10 & \\
Sojabohnen & 5 & 22,5 & 4,8 \\
Sojaschrot & 3 & & \\
Sojaöl & 7 & & \\
Molkenpulver & 5 & & 3,5 \\
Traubenzucker & 5 & 2,5 & 13,4 \\
Kartoffeleiweiß & 5 & 20,0 & 12,2 \\
Rest & & 13,0 & \\
Mineralfutter & 18,5 & & \\
Rohproteingehalt & 14,6 & & \\
Energiegehalt (MJ/kg TS) & TS Trocksto & \\
\hline LVG Le
\end{tabular}

LVG = Lehr- und Versuchsgut Oberschleißheim; TS = Trockensubstanz

Die nachweislich tragenden Sauen (Gruppe 1) wurden in den Wartesauenbereich des Außenklimastalls umgestallt, wo wie im Jungsauenaufzuchtbereich eine individuelle Fütterung an Doppel-Fitmixstationen (Fa. Mannebeck) erfolgte.

Ab dem zehnten Lebenstag erhielten die Ferkel zusätzlich zur Muttermilch ein zugekauftes Ferkelaufzuchtfutter „Schauma Früh“ der Firma Schaumann, Pinneberg. Mit 
dem Absetzen wurde die Fütterung im Flatdeckstall auf betriebseigenes Ferkelfutter umgestellt. Anschließend wurde im Außenklimastall bis zum Erreichen des Testalters sowie im Deckzentrum eine ebenfalls selbst hergestellte Futtermischung für niedertragende Sauen verfüttert. Die Zusammensetzung der einzelnen Futterrationen ist in Tabelle 3 aufgeführt.

\section{Statistische Analyse}

Die statistische Analyse erfolgte mit dem Statistikprogramm SAS 8.02. Für die Varianzanalyse wurde ein gemischtes Modell unter Verwendung von „Proc Mixed“ nach der REML-Methodik (Restricted Maximum Likelihood) genutzt. Folgende Modelle wurden verwendet:

1) Modell für DXA- und LKV-Ergebnisse

$\begin{array}{lll}\mathrm{Y}_{\mathrm{ijkl}} \quad \mu+\text { SauGr }_{\mathrm{i}}+\text { Elternrasse }_{\mathrm{j}} & + \text { Geburtsjahresklasse }_{\mathrm{k}}+\beta \text { (Lebenstage) }+\mathrm{e}_{\mathrm{ijkl}} \\ & = & \text { Beobachtungswert } \\ \mathrm{Y}_{\mathrm{ijkl}} & = & \text { Erwartungswert von y } \\ \mu & = & \text { fixer Effekt der Sauengruppe }(\mathrm{i}=1-3) \\ \text { SauGr }_{\mathrm{i}} & = & \text { zufälliger Effekt der Herkunft }(\mathrm{j}=1-9) \\ \text { Elternrasse }_{\mathrm{j}} & = & \text { zufälliger Effekt der Geburtsjahresklasse }(\mathrm{k}=1-6) \\ \text { Geburtsjahresklasse }_{\mathrm{k}} & = & \text { Kovariable Lebenstage } \\ ß \text { (Lebenstage) } & = & \text { Restfehler (Restvarianz) } \\ \mathrm{e}_{\mathrm{ijkl}} & & \end{array}$

2) Modell für die Fruchtbarkeitsdaten der Sauengruppe 1 (mit Wurf)

$\begin{array}{lll}\mathrm{Y}_{\mathrm{ijk}} \quad \mu+\text { Elternrasse }_{\mathrm{i}} & + \text { Geburtsjahresklasse }_{\mathrm{j}}+\mathrm{e}_{\mathrm{ijk}} \\ & = & \text { Beobachtungswert } \\ \mathrm{Y}_{\mathrm{ijk}} & = & \text { Erwartungswert von y } \\ \mu & = & \text { fixer Effekt der Elternrasse }(\mathrm{i}=1-9) \\ \text { Elternrasse }_{\mathrm{i}} & = & \text { zufälliger Effekt der Geburtsjahresklasse }(\mathrm{j}=1-6) \\ \text { Geburtsjahresklasse }_{\mathrm{j}} & = & \text { Restfehler (Restvarianz). } \\ \mathrm{e}_{\mathrm{ijk}} & & \end{array}$

\section{LKV Ergebnisse}

Ergebnisse und Diskussion

Die Tabelle 4 zeigt die Ergebnisse der Leistungsprüfung und der Zuchtwertschätzung für jede der drei gebildeten Jungsauengruppen (0, 1 und 2). Untersucht wurden Gewicht, Zunahmen, Speckmaß B, Muskelfleischanteil, Fundament, Bemuskelung, Zitzenanzahl, Selektionsindex und Zuchtwert.

In der vorliegenden Studie wurden die drei Jungsauengruppen anhand ihrer Prüfungsergebnisse auf Zuchttauglichkeit und ihrer Fruchtbarkeitsleistung gebildet. Betrachtet man die Ergebnisse der LKV Leistungsprüfung (Tab. 4), so werden erste Unterschiede zwischen den Jungsauengruppen deutlich, die unter anderem zur Bildung der Gruppe 0 geführt haben (Tab. 2).

Vergleicht man die einzelnen Gruppen für die Parameter Zunahmen und Gewicht so zeigt sich, dass die Jungsauengruppe 0 geringere Lebendtageszunahmen und folglich ein deutlich geringeres Lebendgewicht als die Gruppen 1 und 2 aufwies. In beiden Fällen bestand ein signifikanter Unterschied zwischen der Jungsauengruppe 1 und 0 . Das Gewicht und die Tageszunahmen der Gruppe 2 lagen zwar unter denen der 
Gruppe 1, es bestand jedoch keine Signifikanz zwischen den beiden zuchttauglichen Gruppen.

Aus züchterischer Sicht waren die Messergebnisse des Speckmaßes B (SpB) und des Muskelfleischanteils (MFA) von besonderer Bedeutung.

Tabelle 4

Ergebnisse der Leistungsprüfung. Kleinste Quadrate Mittelwerte (LSM) und Standardschätzfehler nach Sauengruppen 0, 1 und 2 (Results of performance testing. Least squares means (LSM) and standard errors of estimation for sow groups 0,1 and 2)

\begin{tabular}{|c|c|c|c|}
\hline Jungsauen & Gruppe 0 & Gruppe 1 & Gruppe 2 \\
\hline Gewicht (kg) & $89,43 \pm 2,68^{\mathrm{ac}}$ & $95,21 \pm 2,25^{b}$ & $92,48 \pm 2,61^{\mathrm{bc}}$ \\
\hline Zunahmen (g) & $494,97 \pm 13,58^{\mathrm{ac}}$ & $523,14 \pm 11,17^{b}$ & $512,39 \pm 13,19^{\mathrm{bc}}$ \\
\hline $\mathrm{SpB}(\mathrm{cm})$ & $8,66 \pm 0,87$ & $8,55 \pm 0.81$ & $8,33 \pm 0,86$ \\
\hline MFA (\%) & $61,46 \pm 1,18^{a^{* c}}$ & $62,13 \pm 1,13^{\text {bc }}$ & $62,43 \pm 1,17^{b}$ \\
\hline Fundament (1-4) & $3.64 \pm 0,1$ & $3,75 \pm 0,06$ & $3,63 \pm 0,09$ \\
\hline Bemuskelung (1-9) & $6,68 \pm 0,26$ & $6,63 \pm 0,24$ & $6,71 \pm 0,26$ \\
\hline Exterieur (1-9) & $6,70 \pm 0,18$ & $6,83 \pm 0,14$ & $6,83 \pm 0,17$ \\
\hline Zitzenanzahl (links) & $6,75 \pm 0,14^{\mathrm{ac}}$ & $6,95 \pm 0,11^{\mathrm{bc}}$ & $7,05 \pm 0,14^{\mathrm{b}}$ \\
\hline Zitzenanzahl (rechts) & $6,76 \pm 0,16$ & $6,96 \pm 0,13$ & $6,84 \pm 0,15$ \\
\hline Index-Wert & $123,25 \pm 15,77$ & $100,99 \pm 10,68$ & $98,66 \pm 15,25$ \\
\hline Zuchtwert & $109,86 \pm 13,42^{\mathrm{bc}}$ & $102,27 \pm 12,98^{\mathrm{ac}}$ & $114,55 \pm 13,37^{b}$ \\
\hline \multicolumn{4}{|c|}{$\begin{array}{l}\text { Gruppe } 0=\text { nicht zugelassen [without mating]; Gruppe } 1=\text { zugelassen, mit Wurf [with litter]; Gruppe } 2=\text { zugelassen, ohne Wurf [mated, } \\
\text { without litter]; SpB = Speckmaß B /back fat depth B]; MFA = Muskelfleischanteil /lean percentage]; Index = Berechnung aus Mastleistung } \\
\text { (Lebenstagszunahme) und Schlachtleistung (Ultraschallmesswerte) korrigiert nach Alter und Gewicht [calculated from daily gain and } \\
\text { ultrasound measurements]; } \\
\text { LSM, die mit unterschiedlichen Buchstaben gekennzeichnet sind, unterscheiden sich signifikant }(\mathrm{p}<0,05) \text {. } \\
\text { LSM marked with different superscripts show significant differences }(p<0.05) ; \mathrm{a}^{*}=\mathrm{p}=0,0501\end{array}$} \\
\hline
\end{tabular}

Bei beiden Parametern hatte die Gruppe 0 die höchsten bzw. niedrigsten Werte mit einem SpB von 8,66 mm und einem MFA von 61,46 \%. Somit schnitt tendenziell die Gruppe 0 als die Gruppe mit dem höchsten Körperfettgehalt (niedrigstem Muskelfleischanteil) ab. Bestätigt wurde diese Tendenz durch den Unterschied des MFA zwischen der Sauengruppe 0 und 2 ( $p=0,0501)$. Bedenkt man aber, dass die Gruppe 0 aufgrund geringer Zuchttauglichkeit oder fehlender Rauscheerscheinungen für zuchtuntauglich erklärt wurde, so lässt sich vermuten, dass allein die erfassten Werte für SpB und MFA nichts über die Zuchttauglichkeit einer Jungsau aussagen.

Subjektiver Einschätzung zufolge war die Gruppe 1 am geringsten bemuskelt und wies somit im Umkehrschluss einen eher höheren Körperfettgehalt auf. Allerdings wurde genau diese Gruppe nach Messungen von SpB und MFA tendenziell magerer als Gruppe 0, jedoch nicht signifikant verschieden zu Gruppe 0 und 2 beurteilt. Zusätzlich zeigte sich, dass Sauen aus der eigenen Untersuchung mit höherem Index- bzw. Zuchtwert nicht zur Zucht zugelassen werden (Gruppe 0), während Sauen mit durchschnittlichem Index und Zuchtwert zumindest einen ersten Wurf produzieren (Gruppe 1). Sowohl Eigenleistungsprüfungsindex als auch Zuchtwert stehen in keiner Beziehung zur späteren Fruchtbarkeit der untersuchten Jungsauen.

\section{DXA-Ergebnisse}

Die ermittelten Werte der DXA-Ganzkörpermessung wie Lebendtageszunahmen (g), Fettanteil (\%), Fettgewebe (g), Magerweichgewebe (g), Knochenmineralgehalt (g) und Knochendichte $\left(\mathrm{g} / \mathrm{cm}^{2}\right)$ sind in Tabelle 5 aufgeführt.

Frühere Untersuchungen, die sich mit der Köperzusammensetzung und Fruchtbarkeit von Jungsauen beschäftigten, bezogen ihre Daten entweder aus Körperanalysen nach der Schlachtung (GAUGHAN et al., 1997; ROZEBOOM et al., 1995; WÄHNER et 
al., 1993; WÄHNER et al., 1995), was aber die Erfassung späterer Wurfdaten unmöglich machte, oder aus Messungen der Rückenspeckdicke bzw. des Muskel-Speck-Verhältnisses (CECHOVA and TVRDON, 2006; GAUGHAN et al., 1997; NEWTON and MAHAN, 1993; PATTERSON et al., 2002; ROZEBOOM et al., 1996; WÄHNER et al., 1995). Rückenspeckdicke und Muskel-Speck-Verhältnis stellen jedoch keine direkten Parameter zur Ermittlung der Körperzusammensetzung dar. Mit Hilfe der Dualenergie-Röntgenabsorptiometrie war es in dieser Studie möglich, die Ganzkörperzusammensetzung in vivo vor der Eingliederung in den Produktionsablauf zu bestimmen und zu bewerten. Da der Scanvorgang eine nicht-invasive Untersuchungsmethode darstellt und somit für die Ganzkörperanalyse eine Schlachtung der zu untersuchenden Tiere nicht mehr notwendig war, konnten die Wurfergebnisse der Gruppe 1-Jungsauen mit erstem Wurf erfasst werden. Nun stellte sich die Frage, inwieweit die einzelnen Jungsauengruppen sich in Bezug auf ihre Körperzusammensetzung voneinander unterschieden. Insbesondere galt es zu klären, ob es überhaupt Unterschiede zwischen den für die Weiterzucht zugelassenen Jungsauen ohne und mit Wurf bzw. den Jungsauen ohne Zuchtzulassung gab.

Tabelle 5

Ergebnisse der Ganzkörperanalyse. Kleinste Quadrate Mittelwerte (LSM) und Standardschätzfehler der DXAMessungen im Ganzkörpermodus für Sauengruppen 0, 1 und 2 (Results from body composition analysis. Least squares means (LSM) and standard errors of estimation from DXA measurements in the whole body modus for sow groups 0,1 and 2)

\begin{tabular}{lccrrrr}
\hline & $\begin{array}{c}\text { LTZ } \\
\mathbf{( g )}\end{array}$ & $\begin{array}{l}\text { Fett } \\
\mathbf{( \% )}\end{array}$ & \multicolumn{1}{c}{$\begin{array}{c}\text { Fett } \\
\mathbf{( g )}\end{array}$} & $\begin{array}{c}\text { MWG } \\
\mathbf{( g )}\end{array}$ & \multicolumn{1}{c}{$\begin{array}{c}\text { BMC } \\
\mathbf{( g )}\end{array}$} & $\begin{array}{c}\text { BMD } \\
\left(\mathbf{g} / \mathbf{c m}^{2}\right)\end{array}$ \\
\hline Gruppe 0 & $478,12^{\mathrm{ac}}$ & $20,57^{\mathrm{a}}$ & $18813^{\mathrm{a}}$ & $64775^{\mathrm{ac}}$ & $2791,35^{\mathrm{ac}}$ & $1,125^{\mathrm{ac}}$ \\
& $\pm 16,65$ & $\pm 1,28$ & $\pm 1304,96$ & $\pm 2225,57$ & $\pm 96,76$ & $\pm 0,024$ \\
\hline Gruppe 1 & $509,92^{\mathrm{b}}$ & $22,22^{\mathrm{b}}$ & $21211^{\mathrm{b}}$ & $67415^{\mathrm{b}}$ & $2979,09^{\mathrm{b}}$ & $1,153^{\mathrm{b}}$ \\
& $\pm 14,66$ & $\pm 1,11$ & $\pm 1107,03$ & $\pm 2051,34$ & $\pm 82,32$ & $\pm 0,022$ \\
\hline Gruppe 2 & $495,77^{\mathrm{bc}}$ & $20,09^{\mathrm{a}}$ & $18765^{\mathrm{a}}$ & $66763^{\mathrm{bc}}$ & $2877,44^{\mathrm{bc}}$ & $1,143^{\mathrm{bc}}$ \\
& $\pm 16,45$ & $\pm 1,28$ & $\pm 1286,32$ & $\pm 2198,66$ & $\pm 95,32$ & $\pm 0,024$ \\
\hline
\end{tabular}

LTZ = Lebendtageszunahmen, MWG = Magerweichgewebe, $\mathrm{BMC}=$ Knochenmineralgehalt, $\mathrm{BMD}=$ Knochenmineraldichte;

LSM, die mit unterschiedlichen Buchstaben gekennzeichnet sind, unterscheiden sich signifikant $(\mathrm{p}<0,05)$.

LSM marked with different superscripts show significant differences $(p<0.05)$.

Die Ergebnisse der Lebendtageszunahmen (LTZ), ermittelt aus DXA Messungen, ähnelten erwartungsgemäß denen der LKV-Zunahmen. Zwischen der Gruppe 0 (478,12 g/Tag) und $1(509,92)$ bestand ein signifikanter Unterschied. Zwar lagen die LTZ der Gruppe $2(495,77)$ unter denen der Gruppe 1, waren statistisch jedoch nicht signifikant verschieden.

Interessant im Vergleich zu den LTZ waren die ermittelten Gesamtkörperfettwerte (Fett, \%, g). Hier bestand ein signifikanter Unterschied nicht nur zwischen den Gruppen 1 (22,22\% bzw. 21211g) und 0 (20,57\% bzw. 18813g), sondern auch zwischen den Gruppen 1 und 2 (20,09\% bzw. 18765g) und das, obwohl die errechneten LTZ (durch DXA und LKV) für diese Gruppen keine Signifikanzen zeigten. Somit ließ sich die Vermutung entkräften, Sauen der Gruppe 2 könnten während ihrer Aufzuchtphase verhaltener gefüttert worden sein als Sauen der Gruppe 1. Unerwartet war, dass die Gruppe 2, die als zuchttauglich beurteilt wurde, durchschnittlich ein halbes Prozent weniger Gesamtkörperfettgehalt bzw. 50 g weniger Gesamtkörperfett aufwies als die zuchtuntauglichen Tiere der Gruppe 0. Bedenkt man jedoch, dass neben der Gruppe 0 auch die Gruppe 2 keinen ersten Wurf produzierte, scheint hier ein direkter Zusammenhang zwischen dem Erreichen und dem Nicht-Erreichen des ersten Wurfes zu be- 
stehen. Aufgrund dieser Ergebnisse kann geschlussfolgert werden, dass Sauen mit einem zu geringen Gesamtkörperfettgehalt nicht oder zumindest schlechter zu einem ersten Wurf kommen. Zu ähnlichen Ergebnissen gelangten auch WÄHNER et al. (1993), die in ihrer Arbeit zu dem Schluss kamen, dass sich ein höherer Fettgehalt im Tierkörper positiv auf die Ovaraktivität auswirkt und damit eine entscheidende Voraussetzung für eine hohe Fruchtbarkeit geschaffen wird. In einer weiteren Untersuchung des Muskel-Speck-Verhältnisses (ermittelt aus Rückenspeckdicke und Muskeldicke) stellten WÄHNER et al. (1995) fest, dass die Trächtigkeitsrate bei fetteren Tieren höher lag als bei magereren. Gleichzeitig zeigten Tiere mit höheren Werten in der Rückenspeckdicke eine größere Anzahl von Follikeln. NEWTON und MAHAN (1993) untersuchten die Reproduktionsleistungen von Jungsauen anhand unterschiedlicher Geburtsgewichtsklassen (120, 135 und 150 kg), erreicht durch verschiedene Futtermischungen. Bei ihnen zeigte eine prozentual höhere Anzahl von Sauen mit geringerem Körpergewicht keine Rausche oder fehlende Konzeption. Eine Aussage, die auch in dieser Studie zutraf. Ebenfalls konform zur vorliegenden Arbeit verliefen Untersuchungen von GAUGHAN et al. (1997), deren Ergebnisse auf Messungen der Rückenspeckdicke basierten. Bei Jungsauen mit mittlerer und hoher Rückenspeckdicke setzte die Pubertät signifikant $(p<0,05)$ früher ein $(170 \mathrm{~d})$ als bei Sauen mit geringerer Rückenspeckdicke (184 d). Des Weiteren zeigten spätere Schlachtergebnisse signifikant $(\mathrm{p}<0,05)$ höhere Fettablagerungen bei Sauen, die in die Pubertät kamen.

PATTERSON et al. (2002) und ROZEBOOM et al. (1995) hingegen trafen eine gegenteilige Aussage. Während PATTERSON et al. (2002) die Auswirkung einer geringeren Wachstumsrate, erzielt durch verschiedene Futtermischungen, auf die sexuellen Abläufe bei Jungsauen untersuchte und zu dem Ergebnis kam, dass Wachstumsleistung oder Gewicht nicht den Beginn der sexuellen Reife bestimmen, untersuchten ROZEBOOM et al. (1995) die Körperzusammensetzung von Jungsauen beim Eintreten der Geschlechtsreife. Sie stellten beträchtliche Variationen in allen Messungen der Körperzusammensetzung (Gewicht, Rückenspeckdicke, Wasser-, Fett-, Protein- und Aschegehalt) fest und kamen zu dem Schluss, dass das Eintreten der Geschlechtsreife weder mit dem Erreichen eines bestimmten Körpergewichtes in Zusammenhang steht, noch mit der Anhäufung einer spezifischen Menge an Körpergewebe.

Magerweichgewebe (MWG), Knochenmineralgehalt (BMC) und Knochenmineraldichte (BMD) zeigten für alle drei Gruppen ein ähnliches Ergebnis. Die höchsten Werte zeigte die Jungsauengruppe 1 gefolgt von der Gruppe 2 und der Gruppe 0, die die niedrigsten Werte aufwies. Für alle drei Parameter bestand ein signifikanter Unterschied zwischen den Gruppen 0 und 1. Die DXA-Knochenmineralergebnisse geben einen Hinweis auf ein besseres Fundament der Gruppe 1 (mit Wurf), gegenüber den Gruppen 0 und 2 (ohne Wurf), wie es durch die Leistungsprüfung ermittelt wurde (Tab. 4).

\section{Fruchtbarkeitsergebnisse}

Für die Auswertung der Fruchtbarkeitsergebnisse wurden nur Tiere der Gruppe 1 erfasst, da nur diese zu einem ersten Wurf gelangten. Tab. 6 zeigt die Ergebnisse der Jungsauen-Gruppe 1 aufgelistet nach unterschiedlichen Elternrassen. Für jeden dieser Genotypen wurden Fruchtbarkeitsparameter, wie Anzahl geborener, totgeborener und aufgezogener Ferkel sowie Ferkelverluste ermittelt. 
Tabelle 6

Kleinste Quadrate Mittelwerte (LSM) und Standardschätzfehler verschiedener Fruchtbarkeitsparameter, getrennt nach unterschiedlichen Genotypen (Elternrassen) [Least squares means (LSM) and standard errors of estimation for different litter performance parameters, separated according sow genotype (parent breeds]

\begin{tabular}{lrrrl}
\hline Elternrasse & Fe geboren & Fe totgeboren & Fe aufgezogen & Fe Verluste \\
\hline DE & $9,38 \pm 0,88$ & $0,25 \pm 0,44^{\mathrm{f}}$ & $8,09 \pm 1,00^{\mathrm{cd}}$ & $1,00 \pm 0,36^{\mathrm{cd}}$ \\
$\mathrm{DL}$ & $9,04 \pm 0,36$ & $0,55 \pm 0,18^{\text {acdf }}$ & $7,89 \pm 0,44^{\mathrm{cd}}$ & $0,55 \pm 0,15^{\mathrm{bc}}$ \\
$\mathrm{Du}$ & $8,92 \pm 0,72$ & $1,25 \pm 0,36^{\mathrm{bf}}$ & $6,78 \pm 0,84^{\mathrm{bd}}$ & $0,83 \pm 0,30^{\mathrm{cd}}$ \\
$\mathrm{Ha}$ & $9,00 \pm 1,77$ & $0 \pm 0,89^{\mathrm{cdf}}$ & $7,94 \pm 1,95^{\mathrm{cd}}$ & $1,00 \pm 0,73^{\mathrm{cd}}$ \\
$\mathrm{Pi}$ & $9,36 \pm 0,67$ & $0,50 \pm 0,34^{\mathrm{df}}$ & $8,52 \pm 0,75^{\mathrm{cd}}$ & $0,29 \pm 0,28^{\mathrm{bc}}$ \\
$\mathrm{SH}$ & $8,67 \pm 1,44$ & $2,33 \pm 0,73^{\mathrm{be}}$ & $5,67 \pm 1,59^{\mathrm{bd}}$ & $0,67 \pm 0,60^{\mathrm{cd}}$ \\
$\mathrm{DL} \times \mathrm{DE}$ & $11,50 \pm 1,25$ & $0,75 \pm 0,63^{\mathrm{bf}}$ & $9,94 \pm 1,40^{\mathrm{ac}}$ & $0,75 \pm 0,52^{\mathrm{cd}}$ \\
Pi x Du & $9,33 \pm 1,44$ & $2,33 \pm 0,73^{\mathrm{b}}$ & $5,94 \pm 1,60^{\mathrm{bd}}$ & $1,00 \pm 0,60^{\mathrm{cd}}$ \\
Pi x Ha & $10,00 \pm 0,79$ & $0,80 \pm 0,40^{\mathrm{bf}}$ & $7,78 \pm 0,91^{\mathrm{cd}}$ & $1,50 \pm 0,33^{\text {ad }}$ \\
\hline
\end{tabular}

DE = Deutsches Edelschwein, DL = Deutsche Landrasse, Du = Duroc, Ha = Hampshire, Pi = Pietrain, SH = Schwäbisch-Hällisches Landschwein, Fe = Ferkel (piglet);

LSM, die mit unterschiedlichen Buchstaben gekennzeichnet sind, unterscheiden sich signifikant $(\mathrm{p}<0,05)$.

LSM marked with different superscripts show significant differences $(p<0.05)$.

Zunächst wurde die Anzahl geborener Ferkel verglichen. Eine besonders hohe Anzahl erreichte die Elternrasse DL x DE mit durchschnittlich 11,5 Ferkeln, gefolgt von der Kreuzung Pi x Ha mit 10 Ferkeln. Die geringsten Ergebnisse erzielten die Genotypen Du $(8,92)$ und SH $(8,67)$. Bei allen anderen Genotypen lag die Wurfgröße zwischen 9 und 9,5 Ferkeln. Zwischen den einzelnen Genotypen traten keine statistisch signifikanten Unterschiede auf, jedoch waren rassenspezifische Unterschiede deutlich erkennbar. Allerdings war die Tierzahl bei den Elternrassen Ha mit nur zwei Tieren, sowie Pi x Du und SH mit drei Tieren sehr gering.

Bei der Anzahl totgeborener Ferkel fiel besonders das Ergebnis der Elternrasse Ha auf, bei denen keine totgeborenen Ferkel auftraten. Ebenfalls eine niedrige Totgeburtenzahl hatten die Rassen DE $(0,25)$, Pi $(0,5)$ und DL $(0,55)$. Hohe Geburtsverlustraten mit 2,33 Ferkeln wiesen hingegen die Sauen der Genotypen Pi x Du und SH auf. Zwischen den genannten Genotypen traten wie in Tabelle 6 ersichtlich, signifikante Unterschiede in der Anzahl totgeborener Ferkel auf.

Die höchste Zahl aufgezogener Ferkel erzielte der Genotyp DL x DE mit 9,94 Ferkeln. Dieser Genotyp erreichte statistisch signifikant bessere Aufzuchtleistungen als die Genotypen Du, Pi x Du und SH. Die schlechtesten Ergebnisse mit unter 6 Ferkeln im ersten Wurf lieferten die Sauengenotypen Pi x Du $(5,94)$ und SH $(5,67)$. Jedoch sind hier die geringen Tierzahlen der Sauengenotypen Pi x Du und SH zu berücksichtigen.

Die Verlustzahl spiegelte die Anzahl Ferkel wieder, die während der Ferkelaufzuchtphase bis zum Erreichen des Absatzgewichtes starben. Bei den Sauengenotypen Pi und DL war die Verlustrate mit 0,29 und 0,55 sehr gering. Die durchschnittliche Verlustzahl für die meisten Gruppen betrug zwischen 0,75 und 1 Ferkel. Nur der Genotyp Pi x Ha lag mit 1,5 Verlustferkeln über diesen Werten. Die statistisch signifikanten Unterschiede zwischen den Genotypen Pi x Ha und Pi bzw. DL unterstrichen dieses Ergebnis.

\section{Vergleich von DXA-Körperfettgehalt und Wurfgröße}

Um Rückschlüsse von der Körperzusammensetzung auf die Wurfgröße ziehen zu können, wurde für alle Jungsauen der Jungsauengruppe 1 die Beziehung zwischen der Anzahl geborener Ferkel und dem DXA-Fettgehalt (\%) in der Abb. 2 dargestellt. Jungsauen mit einem Gesamtkörperfettgehalt von 14-16 Prozent brachten im Durchschnitt zehn Ferkel zur Welt, während die Wurfgröße bei Jungsauen mit $\geq 30$ Prozent Körper- 
fettgehalt mit neun Ferkeln im Durchschnitt um ein Ferkel geringer ausfiel. Gleichzeitig wurde deutlich, dass Jungsauen mit jedem steigenden Prozent Gesamtkörperfettgehalt über 14 Prozent 0,076 Ferkel weniger zur Welt brachten. Obgleich biologisch relevant, war das Ergebnis statistisch nicht signifikant. Es zeigte sich jedoch tendenziell, dass die sehr fetten Sauen mit der Wurfgröße gegenüber den magereren Tieren leicht im Nachteil waren. Untersuchungen von ROZEBOOM et al. (1996) zeigten jedoch keinen großen Effekt der Körperzusammensetzung (gemessen wurden Gewicht und Rückenspeckdicke) auf die Reproduktionsleistungen von Jungsauen. Auch NEWTON und MAHAN (1993) stellten zwischen ihren Geburtsgewichtsklassen keine Unterschiede in der Anzahl lebend- oder totgeborener Ferkel fest. Ergebnisse von WÄHNER et al. (1995) sahen sogar einen Vorteil zu Gunsten der Jungsauen mit einem höheren Körperfettgehalt. Ihre Untersuchung des Muskel-Speck-Verhältnisses ergab eine höhere Anzahl geborener Ferkel bei fetteren Tieren, dies war allerdings nicht signifikant. Ein signifikant ( $<$ 0,05) höheres Ergebnis brachte jedoch der Vergleich lebend geborener Ferkel je 100 Erstbesamungen zwischen Sauen mit mittlerem oder darüber liegenden Muskel-Speck-Verhältnis gegenüber Tieren mit sehr geringem Fettanteil im Körper. Eine ähnliche Aussage trafen GAUGHAN et al. (1997), indem sie feststellten, dass Jungsauen mit einer geringen Rückenspeckdicke signifikant weniger Follikel (durchschnittlich 13) aufwiesen, als Tiere mit mittlerer (19 Follikel) oder hoher (18 Follikel) Rückenspeckdicke. Auch CECHOVA und TVRDON (2006), die die Beziehung zwischen der Rückenspeckdicke und den Reproduktionsmerkmalen bei Tschechischen Large White Sauen untersuchten, kamen in ihrer Arbeit zu dem Ergebnis, dass Sauen mit einer größeren Rückenspeckdicke Würfe mit einer höheren Anzahl lebend geborener und abgesetzter Ferkel produzierten.

Eine Sonderstellung im Vergleich zu den in dieser Studie ermittelten Fruchtbarkeitsergebnissen europäischer bzw. amerikanischer Schweinerassen nehmen chinesische Schweinerassen ein, die einen sehr hohen Körperfettgehalt und gleichzeitig eine besonders hohe Fruchtbarkeit aufweisen. In einer Untersuchung von WHITE et al. (1993) wurden Fruchtbarkeitsparameter von chinesischen Meishan- und europäischen Yorkshire-Jungsauen verglichen. Die Sauen der Rasse Meishan erzielten mit durchschnittlich 12,4 Ferkeln eine signifikant $(p<0,05)$ höhere Wurfgröße als die Sauen der Rasse Yorkshire mit 7,4 Ferkeln. Zu dem gleichen Ergebnis gelangten GALVIN et al. (1993). Sie stellten signifikante Unterschiede $(\mathrm{p}<0,05)$ in der Anzahl von Embryonen am 20.-22. Tag der Trächtigkeit zwischen Meishan-Sauen (23,4 Embryonen) und europäischen Large-White-Sauen (15,2 Embryonen) fest. YOUNG (1995) untersuchte die Reproduktionsleistungen von F1-Jungsauen die zu 50\% chinesische Rasseanteile (Meishan, Fengjing, Minzhu) oder Duroc und zu 50\% europäische Landrasse enthielten. Er gelangte zu dem Ergebnis, dass alle drei chinesische Kreuzungsrassen signifikant $(\mathrm{p}<0,05)$ größere Würfe hatten als die Duroc-Kreuzungen. Eine weitere Analyse von YOUNG (1998) zeigte, dass Kreuzungssauen die zu 1/4 chinesische Rasseanteile (Meishan, Fengjing, Minzhu) enthielten, ebenfalls größere Würfe hatten als Jungsauen mit 1/4 Duroc-Rasseanteil. Allerdings trat nur zwischen den Kreuzungsrassen Fengjing und Duroc ein signifikanter Unterschied $(p<0,05)$ auf. 


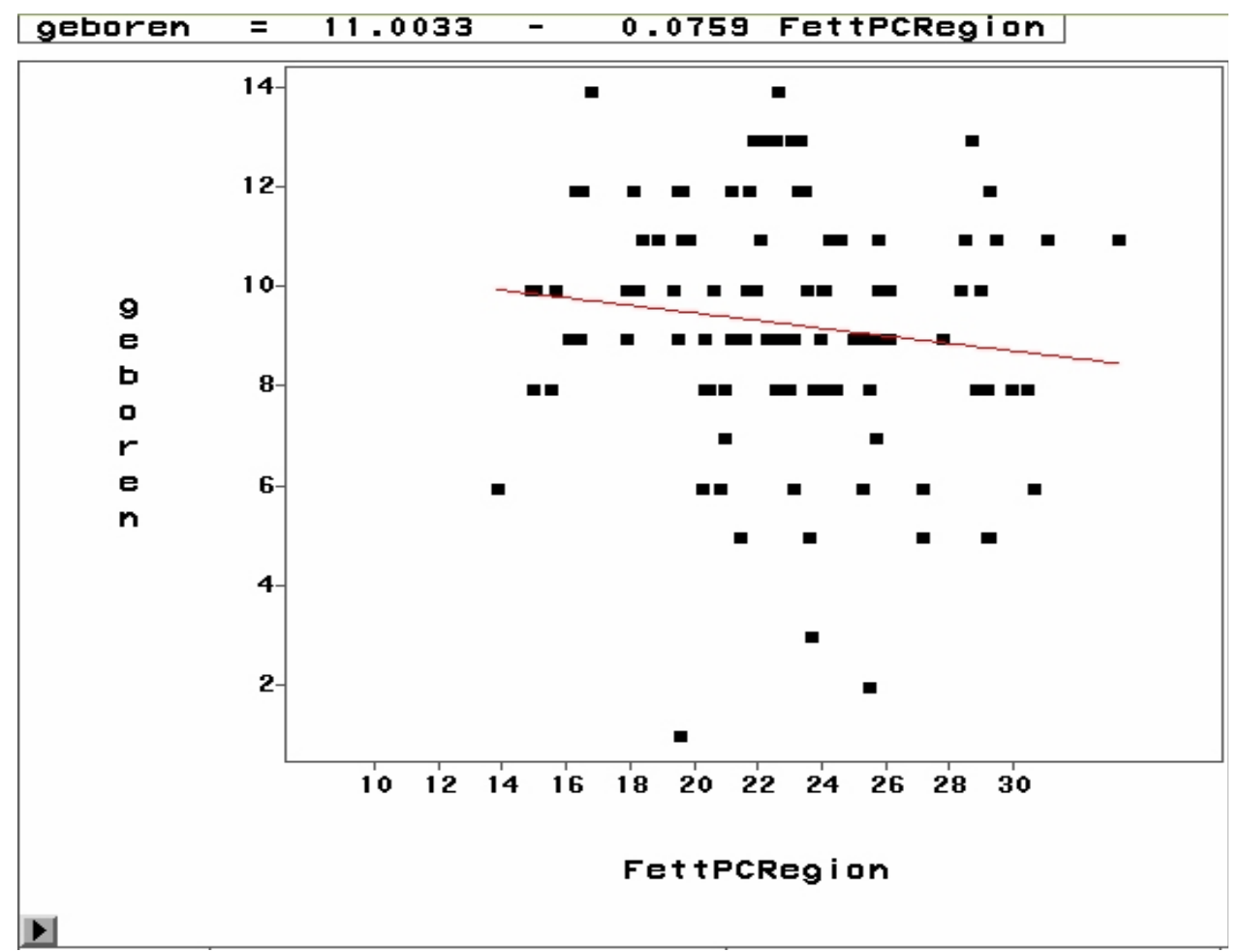

$\mathrm{r}=0,12 ; \mathrm{p}=0,17$; geboren $=$ Anzahl geborener Ferkel; FettPCRegion = prozentualer Körperfettgehalt

Abb. 2: Beziehung zwischen Anzahl geborener Ferkel und DXA-Fett (\%) von Jungsauen (Relation between number of piglets born and DXA fat (\%) of gilts)

Der Zusammenhang zwischen dem individuellen Körperfettgehalt von Jungsauen und der Wurfgröße ist züchterisch von besonderem Interesse. In der vorliegenden Untersuchung fiel auf, dass Tiere der Rasse Schwäbisch-Hällisches Landschwein einen sehr hohen Gesamtkörperfettgehalt (27,16\%) aufwiesen und die Rasse Duroc sehr mager $(18,75 \%)$ war. Genau diese beiden Rassen hatten die geringsten Wurfgrößen, während Sauen mit mittlerem Gesamtkörperfettgehalt die besten Wurfergebnisse erzielten. Diese Aussage ähnelt den bereits erwähnten Untersuchungsergebnissen von GAUGHAN et al. (1997). WÄHNER et al. (2001) bzw. JOHN et al. (2001) untersuchten den Einfluss der Wachstumsintensität (gemessen durch Lebendtageszunahmen) und der Seitenspeckdicke auf die Fruchtbarkeits- und Aufzuchtleistungen von Jungsauen. Sie kamen in ihrer Arbeit zu dem Ergebnis, dass Jungsauen mit mittleren Lebendtageszunahmen die besten Reproduktionsergebnisse erzielten, während Jungsauen mit sehr hohen Zunahmen generell geringere Aufzuchtleistungen erbrachten. Außerdem zeigte sich ein positiver Effekt einer zunehmenden Seitenspeckdicke auf die Reproduktionsergebnisse. Allerdings wirkte sich eine Überkonditionierung der Jungsauen bis zum Besamungszeitpunkt negativ auf die Reproduktionsleistung aus. NEWTON und MAHAN (1993) stellten fest, dass sich die Ferkelsterblichkeit mit steigendem Geburtsgewicht signifikant $(\mathrm{p}<0,01)$ erhöht. Auch in Bezug auf die Ferkelsterblichkeit (totgeborene Ferkel + Ferkelverluste) lag das Schwäbisch-Hällische Landschwein mit durchschnittlich drei Ferkeln pro Wurf an der Spitze aller untersuchten Sauengenotypen. Inwieweit sich ein „idealer Körperfettgehalt“ bei Jungsauen in Abhängigkeit von der Rasse eingrenzen lässt, sollte in weiterführenden Untersuchungen geprüft werden. 
BÖTTINGER, M.:

\section{Literatur}

Untersuchung des Einflusses verschiedener Produktionssysteme auf das Wachstum beim Schwein unter Verwendung der Dualenergie-Röntgenabsorptiometrie zur Messung von Fett, Magerweichgewebe und Knochenmineralansatz. Diss., Tierärztliche Fakultät, Ludwig-Maximilians-Universität München, 2005

CECHOVA, M.; TVRDON, Z.:

Relationships between back fat thickness and parameters of reproduction in Czech Large White sows. Arch. Tierz., Dummerstorf 49 (2006), 363-369

GALVIN, J.M.; WILMUT, I.; DAY, B.N.; RITCHIE, M.; THOMSON, M.; HALEY, C.S.:

Reproductive performance in relation to uterine and embryonic traits during early gestation in Meishan, large white and crossbred sows. J. Reprod. Fertil., 98 (1993) 2, 377-384

GAUGHAN, J.B.; CAMERON, R.D.; DRYDEN, G.M.; YOUNG, B.A.:

Effect of body composition at selection on reproductive development in large white gilts. J. Anim. Sci., 75 (1997), 1764-1772

JOHN, A.; WÄHNER, M.; HOFFMEYER, C.:

Zum Einfluss des Wachstums und der Seitenspeckdicke auf die Fruchtbarkeits- und Aufzuchtleistung von Jungsauen. 2. Mitteilung: Beziehung zwischen den Merkmalen und Diskussion. Arch. Tierz., Dummerstorf 44 (2001), 277-290

KOO, W.W.; HAMMAMI, M.; HOCKMAN, E.M.:

Use of fan beam dual energy x-ray absorptiometry to measure body composition of piglets. J. Nutr., 123 (2002), 1380-1383

LUKASKI, H.C.; MARCHELLO, M.J.; HALL, C.B.; SCHAFER, D.M.; SIDERS, W.A.: Soft tissue composition of pigs measured with dual x-ray absorptiometry: comparison with chemical analyses and effects of carcass thicknesses. Nutrition, 15 (1996), 697-703

MITCHELL, A.D.; CONWAY, J.M.; SCHOLZ, A.M.:

Incremental changes in total and regional body composition of growing pigs measured by dual-energy x-ray absorptiometry. Growth Dev. Aging, 60 (1996), 95-105

MITCHELL, A.D.; SCHOLZ, A.M.:

Dual-energy x-ray absorptiometry (DXA) analysis of growth and composition of pigs of different ryanodine receptor genotypes. Arch. Tierz., Dummerstorf 40 (1997), 47-56

MITCHELL, A.D.; SCHOLZ, A.M.; CONWAY, J.M.:

Body composition analysis of pigs from 5 to $97 \mathrm{~kg}$ by dual-energy x-ray absorptiometry. Appl. Radiat. Isot., 49 (1998), 521-523

MITCHELL, A.D.; SCHOLZ, A.M.; PURSEL, V.G.:

Dual-energy X-ray absorptiometry measurements of the body composition of pigs of 90- to 130 kilograms body weight. Ann. N.Y. Acad. Sci., 904 (2000), 85-93

MITCHELL, A.D.; SCHOLZ, A.M.:

Techniques for Measuring Body Composition of Swine. (Chapter 41). In: Swine Nutrition $2^{\text {nd }}$ Edition (Ed.: A.J. Lewis and L. Lee Southern), CRC Press, Boca Raton, FL, USA (2001), 917-960

MITCHELL, A.D.; SCHOLZ, A.M.; PURSEL, V.G.:

Prediction of the in vivo Body Composition of Pigs Based on Cross-Sectional Region Analysis of Dual Energy X-Ray Absorptiometry (DXA) Scans. Arch. Tierz., Dummerstorf 45 (2002), 535-545

MÜLLER, S.; POLTEN, D.C.:

Vergleichsuntersuchungen zur Ultraschall-Speckdickenmessung beim Schwein im Rahmen der Eigenleistungsprüfung. Arch. Tierz., Dummerstorf 47 (2004), 249-263

NEWTON, E.A.; MAHAN, D.C.:

Effect of initial breeding weight and management system using a high-producing sow genotype on resulting reproductive performance over three parities. J. Anim. Sci., 71 (1993), 1177-1186

PATTERSON, J.L.; BALL, R.O.; WILLIS, H.J.; AHERNE, F.X.; FOXCROFT, G.R.: The effect of lean growth rate on puberty attainment in gilts. J. Anim. Sci., 80 (2002), 1299-1310

PIETROBELLI, A.C.; FORMICA, C.; WANG, Z.; HEYMSFIELD, S.B.: Dual-energy X-ray absorptiometry body composition model: review of physical concepts. Am. J. Physiol., 271 (1996), E941-E951

ROZEBOOM, D.W.; PETTIGREW, J.E.; MOSER, R.L.; CORNELIUS, S.G.; EL KANDELGY, S.M.: Body composition of gilts at puberty. J. Anim. Sci., 73 (1995), 2524-2531

ROZEBOOM, D.W.; PETTIGREW, J.E.; MOSER, R.L.; CORNELIUS, S.G.; EL KANDELGY, S.M.: Influence of gilt age and body composition at first breeding on sow reproductive performance and longevity. J. Anim. Sci., 74 (1996), 138-150

SCHNURRBUSCH, U.:

Bedeutung des Körperfettes für die Fruchtbarkeit von Sauen. Großtierpraxis, 6 (2005) 11, 6-12

SCHOLZ, A.M.: 
In-vivo-Methoden zur Analyse von Muskelstoffwechsel und Körperzusammensetzung beim Schwein unter besonderer Berücksichtigung genetischer Einflüsse. Habilitationsschrift, Institut für Tierzucht der Ludwig-Maximilians-Universität München, 2002

SCHOLZ, A.M.; SOFFNER, P.; LITTMANN, E.; PESCHKE, W.; FÖRSTER, M.:

Genauigkeit der Dualenergie-Röntgenabsorptiometrie (DXA) zur Ermittlung der Schlachtkörperzusammensetzung von Schweinehälften (kalt, 30-39 kg) anhand der EU Referenzzerlegung. Züchtungskunde, 74 (2002), 376-391

SVENDSEN, O.L.; HAARBO, J.; HASSAGER, C.; CHRISTIANSEN, C.:

Accuracy of measurements of body composition by dual-energy x-ray absorptiometry in vivo. Am. J. Clin. Nutr., 57 (1993), 605-608

WÄHNER, M.; ENGELHARDT, S.; SCHNURRBUSCH, U.; GOTTSCHALK, J.; SCHARFE, St.;

PFEIFFER, H.:

Beziehung zwischen dem Fleisch-Fettansatz und den Konzentrationen von 17- $\beta$-Östradiol bzw. Progesteron in der Follikelflüssigkeit sowie im Muskel- und Fettgewebe bei weiblichen Schweinen. Züchtungskunde, 65 (1993), 382-393

WÄHNER, M.; ENGELHARDT, S.; SCHNURRBUSCH, U.; PFEIFFER, H.:

Beziehung zwischen Kriterien des Fleisch- bzw. Fettansatzes und den 17ß-Östradiol- bzw. Progesteronkonzentrationen in der Follikelflüssigkeit, im Muskel- und Fettgewebe, der Ovulationspotenz sowie der Fruchtbarkeitsleistung von Jungsauen. Arch. Tierz., Dummerstorf 38 (1995), 187-197

WÄHNER, M.; JOHN, A.; HOFFMEYER, C.:

Zum Einfluss des Wachstums und der Seitenspeckdicke auf die Fruchtbarkeits- und Aufzuchtleistung von Jungsauen. 1. Mitteilung: Vergleich der Merkmale Wachstum, Seitenspeckdicke, Fruchtbarkeit und Aufzuchtleistungen. Arch. Tierz., Dummerstorf 44 (2001), 157-166

WHITE, B.R.; MCLAREN, D.G.; DZIUK, P.J.; WHEELER, M.B.:

Age at puberty, ovulation rate, uterine length, prenatal survival and litter size in Chinese Meishan and Yorkshire females. Theriogenology, 40 (1993), 85-97

YOUNG, L.D.:

Reproduction of F1 Meishan, Fengjing, Minzhu and Duroc gilts and sows. J. Anim. Sci., 73 (1995), 711-721

YOUNG, L.D.:

Reproduction of $3 / 4$ White Composite and $1 / 4$ Duroc, $1 / 4$ Meishan, $1 / 4$ Fengjing or $1 / 4$ Minzhu gilts and sows. J. Anim. Sci., 76 (1998), 1559-1567

Eingegangen: 18.08.2006

Akzeptiert: 24.10.2006

Anschrift der Verfasser

HENNING HOFFSCHULTE, Priv.-Doz. Dr. ARMIN M. SCHOLZ*

Lehr- und Versuchsgut der Tierärztlichen Fakultät der Ludwig-Maximilians-Universität

München

Sankt Hubertusstraße 12

D-85764 OBERSCHLEIßHEIM

*Autor für Korrespondenz, E-Mail: Armin.Scholz@lvg.vetmed.uni-muenchen.de 